DOCTRINA

\title{
Rediseñando la titularidad de las obras: Inteligencia artificial y robótica
}

\author{
Redesigning the ownership of artworks: \\ Artificial and robotic intelligence \\ Ana Karin Chávez Valdivia \\ Universidad La Salle de Arequipa, Perú
}

RESUMEN Nos atrevemos a sostener que no existe campo de estudio o área de conocimiento en que no haya incursionado la dupla de robótica e inteligencia artificial; y que en la actualidad uno de los principales cuestionamientos gira en torno a la posibilidad de otorgar una personalidad jurídica específica a determinados tipos de robots $y$, en consecuencia, el reconocimiento de la titularidad sobre ciertos derechos. En este sentido, una interrogante aún sin resolver aborda la pregunta sobre quién es el propietario de las obras creadas por la inteligencia artificial. Más allá de una titularidad «sobreentendida», el estado actual de las creaciones hace necesario un serio análisis respecto de las diversas posibilidades revestidas de un carácter de negación absoluta o duda razonable.

PALABRAS CLAVE Robots, inteligencia artificial, personalidad jurídica, arte, derechos de autor.

ABSTRACT We dare to affirm there is no area of knowledge where robotic-artificial intelligence duo has not entered yet; and that currently one of the main questions revolves around the possibility of granting a specific legal personality to certain kind of robots and consequently the conferring of ownership over certain rights; in this sense, an unresolved question is who owns the artworks created by artificial intelligence. Beyond an "implied" ownership, the current state of creations makes it necessary to execute a serious analysis related to the varying possibilities still covered by a character of absolute negation or reasonable doubt.

KEYWORDS Robots, artificial intelligence, legal personality, art, copyright. 


\section{Introducción}

Aunque la palabra robot es un concepto popular del siglo XX, en realidad forma parte de una historia mucho más larga. Podría decirse que desde sus inicios, los robots han estado estrechamente entrelazados con el arte, lo que se evidencia sobre todo en que el término en sí mismo deriva de un contexto teatral y que, en el transcurso de los años, su presencia en películas de ciencia ficción y en la literatura en general sigue siendo clara. Por otro lado, es innegable que existen ciertas relaciones intermedias entre la robótica y el arte, como las asociadas a la innovación y el utilitarismo en la ingeniería robótica o las expresiones sociales y culturales en las artes.

Podríamos afirmar que una de las razones por las cuales la dupla de robótica e inteligencia artificial genera polémica y hasta cierto rechazo encuentra sustento en temores de diversa naturaleza: tal vez el principal es la inminente posibilidad de convertirse en una amenaza a la subsistencia humana. Sin embargo, es innegable que las implicancias y efectos de esta conjunción ya han comenzado a revolucionar las estructuras existentes y significarán innumerables desafíos regulatorios que los actuales marcos legales - en su mayoría - no están preparados para enfrentar. De manera contradictoria, esta tecnología disruptiva se ha convertido no sólo en un factor esencial, sino en gran medida indispensable para el despliegue de prácticamente todas nuestras actividades habituales.

El presente estudio considera, en primer lugar, la revisión de diversas acepciones como parte del proceso de evolución de la robótica y la inteligencia artificial. Tras recoger el análisis doctrinal en torno a dos personalidades jurídicas diferenciadas, analiza y plantea la procedencia de una personalidad jurídica específica para los robots, lo que conlleva al obligado análisis en torno a si estos pueden o deben tener derechos.

En segundo lugar, intenta responder a la siguiente interrogante: ¿Quién es el propietario de las obras creadas por la inteligencia artificial? Para ello, la aborda desde distintas ópticas, por lo que se analiza la creatividad en relación con la creación de obras, así como la titularidad de los derechos de autor o reconocimiento de estos derechos a creadores no humanos.

Por último, nuestra investigación recoge y analiza diversos tratamientos legislativos internacionales en torno al tema, enfatizando la necesidad de implementación de principios y regulaciones o del replanteamiento de los ya existentes.

\section{Robótica e inteligencia artificial: Precisiones imprecisas}

Durante todos estos años, la casi totalidad de leyes existentes se ha centrado sólo en los seres humanos. El desarrollo de la inteligencia artificial, a la par de haber revolucionado los múltiples entornos en los que ha incursionado, ha traído consigo diversos y novedosos problemas, que los sistemas legales actuales en su gran 
mayoría están parcial o mínimamente equipados, mientras que otros sencillamente no lo están.

Así, surgen interrogantes de toda índole respecto de las posibles implicancias jurídicas como resultado de los despliegues de la inteligencia artificial y la robótica en diferentes escenarios y contextos. Mientras que de un lado mucho se viene comentando y escribiendo sobre posibles problemas que como sociedad tendremos que afrontar a corto, mediano y largo plazo, del otro encontramos grandes expectativas, promesas y visiones de un futuro ideal que se harán viables precisamente gracias a esta tecnología.

Consideramos que la discusión no debe girar sobre si seremos desplazados, si nuestros despliegues estarán condicionados por organismos cibernéticos o si finalmente el mundo llegará a ser controlado por androides. El punto crucial y determinante ahora es establecer la forma en que la humanidad y la inteligencia artificial podrán coexistir en armonía y sin temor, en beneficio de todos los seres humanos $y$, por qué no decirlo desde ahora, en beneficio también de los propios androides, sea cual fuere su tipología.

Nos encontramos en un punto de quiebre en torno al tema, debido a que ya se han asumido radicales posturas optimistas, pesimistas y pragmáticas. Más allá de encontrar un bando aliado a nuestros temores, ilusiones o incertidumbres, toca enfocar nuestra atención y esfuerzos hacia la forma en que la humanidad y la inteligencia artificial deben empezar a interactuar.

Parece ser indiscutible que la cada vez mayor incursión de robótica y la inteligencia artificial se perfila como una nueva forma de dependencia. Así sucedió con internet, teléfonos celulares y otras tecnologías análogas. De ahí la importancia de tratar algunas precisiones en torno a la conceptualización que se tiene de un robot y de la inteligencia artificial, aun cuando, incluso entre los expertos, hay poco acuerdo cuando se trata de definir, caracterizar o incluso identificar qué es o qué no es un robot o una inteligencia artificial.

Illah Nourbakhsh (2013: 14) considera que la robótica moderna trata sobre cómo algo puede percibir el mundo, dar sentido a su entorno, para luego empujar al mundo de regreso y hacer el cambio. Señala al mismo tiempo que nunca hay que preguntar a un robotista qué es un robot, ya que la respuesta cambia demasiado rápido. Cuando los investigadores terminan su debate más reciente sobre qué es y qué no es un robot, la frontera avanza a medida que nacen nuevas tecnologías de interacción. Por otro lado, precisa el autor que hay una cualidad especial de la robótica moderna que es muy relevante para la manera en que nuestro mundo está cambiando: los robots son una nueva forma de pegamento vivo entre nuestro mundo físico y el universo digital que hemos creado. Los robots tienen sensores físicos y motores: pueden operar en el mundo real tan bien como cualquier programa de software opera en internet. Estarán incorporados en nuestros espacios físicos y tendrán sus propias mentes gracias a la 
inteligencia artificial. Señala en forma acertada Nourbakhsh (2013: 15) que los robots también están completamente conectados con el mundo digital: son mucho mejores navegando, compartiendo información y participando en el mundo en línea que los humanos. Señala que se ha inventado una nueva especie, parte material y parte digital, que eventualmente tendrá cualidades sobrehumanas en ambos mundos a la vez, por lo que, en su opinión, las preguntas que nos quedan son cómo compartiremos nuestro mundo con estas nuevas criaturas, y cómo cambiará esta nueva ecología a quienes somos ahora y a la forma en cómo actuamos. Estas interrogantes sin duda abren la posibilidad a innumerables, cuestionables e incompletas respuestas, así como a diversas consideraciones en torno a su verdadera dimensión. En este contexto, afirma Nourbakhsh (2017: 46) que las tecnologías nos darán un nuevo potencial, y sostiene que en lugar de reaccionar caso por caso a lagunas legales reveladas por máquinas cada vez más ingeniosas, la jurisprudencia debería recoger de antemano la experiencia y los medios necesarios para producir futuros escenarios robóticos.

John Jordan (2015: 11-12), por su parte, admite abiertamente que ya existe una considerable indeterminación y deslizamiento en la terminología, al tiempo que «los límites del campo de juego conceptual», abordados por el autor, no sólo fueron definidos por la ciencia ficción, sino que ésta se convirtió en la fuente original del término. La palabra robot llegó al mundo a través de la obra teatral de Karel Čapek en 1920, que usó para nombrar una clase de sirvientes o trabajadores artificiales. En checo, como en varias otras lenguas eslavas, la palabra robota - o alguna variación de ésta- denota «servidumbre o trabajo forzoso». ${ }^{1}$ Desde la publicación de la obra de Čapek, los robots se infiltraron en el espacio de la ficción, aunque lo que constituye con exactitud un robot difiere y admite una amplia variedad de formas, funciones y configuraciones.

Brian David Johnson (2011: 44) señaló que la ciencia ficción nos permite vernos a nosotros mismos bajo una nueva luz, a la luz de un nuevo futuro, uno que no es el nuestro, sino que refleja directamente quiénes somos y hacia dónde podríamos dirigirnos.

Por su parte, Gunkel (2018: 29) considera que, además de influir en los programas de investigación y desarrollo, la ciencia ficción también ha demostrado ser un mecanismo muy conveniente - quizás incluso el mecanismo preferido- para examinar las oportunidades sociales y los desafíos de la innovación tecnológica en inteligencia artificial y robótica. Señala el autor que las definiciones sobre el término que pueden hallarse en diccionarios por lo general son demasiado amplias, en la medida en que podrían aplicarse a cualquier programa informático; y a su vez, demasiado limitadas, porque tienden a privilegiar formas y configuraciones similares a las humanas que,

1. Karel Čapek no fue, sin embargo, el creador del término, que es atribuido a su hermano mayor, el pintor Josef Čapek. 
más allá de lo que se describe en la ciencia ficción, son más la excepción que la regla (Gunkel, 2018: 32).

Es indiscutible que, en cualquier forma en que hayan aparecido - ya sea en la ciencia ficción o, mucho después, en la práctica de ingeniería real一, se establecieron expectativas para lo que un robot es o puede ser. Antes de que los ingenieros hayan tratado de desarrollar prototipos de trabajo, escritores, artistas y cineastas habían imaginado qué hacen o pueden hacer los robots, qué configuraciones podrían tomar y qué problemas podrían generar a las personas y comunidades humanas.

Neil M. Richards y William D. Smart (2013: 4), abordaron desde el punto de vista legal la pregunta de qué es un robot. Encontraron que, para la gran mayoría de personas, la respuesta está informada por lo que ven en las películas, los medios populares $y$, en menor medida, en la literatura; por tanto, las personas sacan conclusiones a partir de las representaciones de robots que han visto a través de estos medios, debido a que pocas han visto un robot real.

Los autores conceptualizan al robot como un sistema construido que muestra agencia física y mental, pero que no está vivo en el sentido biológico (Richards y Smart, 2013: 5). Es decir, un robot es una máquina fabricada que se mueve por el mundo, y parece tomar decisiones racionales sobre qué hacer. Señalan, además, que es importante tener en cuenta que la atribución de agencia es subjetiva, es decir, el sistema sólo debe parecer que tiene agencia ante un observador externo para cumplir con sus criterios. Además, precisan que su definición excluye a las inteligencias artificiales totalmente basadas en software que no ejercen ninguna agencia en el mundo físico. Así, su definición intencionalmente deja abierto el mecanismo que causa la aparente agencia. Consideran también que el sistema puede ser controlado por un software informático inteligente o teleoperado por un operador humano remoto. $\mathrm{Si}$ bien ambos sistemas son robots, según su definición, plantean que las implicaciones legislativas para cada uno de ellos serían bastante diferentes.

Por otro lado, destacan algunos temas comunes que en su opinión parecen estar surgiendo. Abordan en primer lugar que muchos robots de investigación ahora son multipropósito en lugar de estar diseñados para una sola tarea. En segundo lugar, los están comenzando a interactuar con personas que no saben nada sobre robots; si bien esto es necesario para alcanzar el potencial a largo plazo de la tecnología, también complica las cosas, ya que los humanos son impredecibles, fáciles de dañar y difíciles de complacer, por lo que en la actualidad muchas investigaciones están dirigidas a lograr que los robots los manejen con gracia y seguridad. En tercer lugar, señalan que a medida que se resuelven los desafíos técnicos subyacentes de la percepción y el razonamiento, los robots se están volviendo cada vez más autónomos. Por último, refieren que hay un enfoque creciente en los robots que funcionan en el mundo real, no sólo en el laboratorio. Esto requiere que lidiamos con toda la incertidumbre e imprevisibilidad inherentes al mundo en que vivimos (Richards y Smart, 2013: 11). 
En este contexto, los autores consideran que a medida que los robots se vuelven más multipropósito, será difícil imaginar a priori cómo serán utilizados y, por lo tanto, crear protecciones legislativas y de consumo integrales para ellos. En el momento en que un robot pueda hacer todo lo que un humano puede hacer, existirán pocos límites prácticos respecto del uso que se puede dar a un robot, por lo que se cuestionan válidamente cómo se legislará tal sistema, y sostienen que será complicado. Continúan su análisis señalando que, a medida que los robots ingresen a la vida pública y a nuestros hogares privados, las protecciones asociadas con ellos deberán ser más completas y robustas que las implementadas hoy para los robots de investigación. En este orden de ideas, diversos estudios enfocan su atención en torno a la privacidad (Rueben y otros, 2017: 425; 2019: 436; Balali y otros, 2019: 720). Creemos que esos resultados conducen y facilitan el análisis y la propuesta en torno al tratamiento diferenciado por su propia naturaleza- de los denominados «robots sociales», ya que en la medida en que se vuelvan más autónomos, la cuestión de dónde descansa la responsabilidad cuando algo sale mal se complica, y su ingreso al mundo real hace que nuestra capacidad de predecir lo que sucederá disminuya drásticamente, por lo que el diseño de una legislación que sea integral, pero que no limite demasiado el uso de estos sistemas, será un desafío. Más aún, cuando ya no se considere tan sólo a los sistemas que son claramente autónomos o teleoperados, sino también a la nueva generación de sistemas de autonomía compartida.

En respuesta a las imprecisiones existentes, organizaciones profesionales, autores y manuales sobre robótica ofrecen lo que se pretende sea una caracterización más precisa. Para Bekey, citado por Gunkel (2018: 33); un robot es una máquina que cumple las funciones sense-think-act (percibir, pensar y actuar), por lo que un robot debe tener sensores, capacidad de procesamiento que emule algunos aspectos de la cognición y «actuadores». Por otro lado, para Jones y Millar (2017: 598) no existe una definición concisa e indiscutible de lo que es un robot y puede entenderse mejor a través del paradigma sense-think-act, que permite distinguir a los robots como cualquier tecnología que recopila datos sobre el medio ambiente a través de uno o más sensores, procesa la información de manera relativamente autónoma y actúa en el mundo físico.

Al mismo tiempo, debemos aceptar que pocas tecnologías complejas tienen una definición única, estable e incontestada, y los robots no son la excepción. Refiere Calo (2015: 529) que existe cierto consenso sobre la idea de que los robots son objetos mecánicos que absorben el mundo, procesan lo que sienten y a su vez actúan sobre el mundo, y resalta que la utilidad aquí del paradigma sense-think-act radica en distinguir a los robots de otras tecnologías, ya que la idea de un robot o sistema robótico es que combine los tres.

Por su parte, Winfield (2012: 8) ofrece una lista de características de calificación para refinar la definición y delimitar con mayor precisión qué es y qué no es un robot; y aunque en apariencia sería otra versión de sense-think-act, Winfield agrega una 
calificación importante: embodied, lo que deja en claro que un bot de software, un algoritmo o implementaciones de inteligencia artificial como Watson o AlphaGo no son robots en sentido estricto, según su planteamiento.

Cabe mencionar, sin embargo, que esta lista no agota todas las diferentes formas en que se ha definido, explicado o caracterizado el robot. En consideración de John Jordan (2015: 11), estas definiciones están aún sin resolver, incluso entre las que se consideran más expertas en el campo.

A esta línea de pensamiento se aúna Ryan Calo (2015: 531), quien considera que un sistema actúa sobre su entorno en la medida en que cambia ese entorno de manera directa. Una tecnología no actúa, y por lo tanto no es un robot, cuando sólo proporciona información en un formato inteligible, ya que debe ser de alguna manera. Un robot en el sentido más fuerte y completo del término existe en el mundo como un objeto corporal con la capacidad física de un despliegue propio. Así, es de particular interés para el análisis legal una definición funcional de lo que significa que la tecnología actúe, en lugar de que sólo informe.

En este contexto, Calo señala que para hablar de una tecnología transformadora - por la combinación que implica- debe tenerse presentes las cualidades esenciales que la caracterizan como tal y que permiten ir más allá de una sola definición técnica de lo que sería un robot. Sostiene el autor que, ya sea que estas cualidades se presenten de manera independiente o en combinación, resultan relevantes para una variedad extraordinariamente amplia de contextos legales. Consideramos necesario referirlas en el mismo idioma en que fueron propuestas por el autor: embodiment, emergence y social valence. Esto reviste trascendental importancia para entender el sentido de su estudio, ya que las interpretaciones o traducciones podrían resultar no sólo un tanto engañosas, sino también inducirnos a error respecto del real sentido de estas cualidades esenciales, más aún si las consideramos relevantes en el intento de establecer una nueva personalidad jurídica y subsecuentes derechos.

En este orden de ideas, y en consideración al estado actual de la cuestión, nos alineamos a las posturas señaladas por Winfield, Richards, Smart y Calo. Consideramos que la antropomorfización - al menos por ahora- desempeñará un rol trascendental en torno al establecimiento y determinación de instituciones integrantes de un posible sistema legal tuitivo para los sistemas robóticos inteligentes, entre ellos la personalidad jurídica, con todo lo que esto implique.

Por su parte, refieren Groom y otros (2009: 32) que el grado de antropomorfismo en robots afecta la manera en que las personas interactúan con ellos. Precisan que la investigación ha demostrado que se elogia más a los robots antropomórficos y se los castiga menos en las interacciones colaborativas entre el equipo humano y el robot. Que las formas más antropomórficas de robots también aumentan los sentimientos de utilidad y comprensión, y que se percibe que son más comprensivas, amigables e inteligentes que los robots funcionales. 
Cobra importancia señalar entonces que los robots también evocan respuestas en las personas. Calo (2015: 549) refiere que el sicólogo Peter Kahn y sus colegas llevaron a cabo una serie de experimentos para intentar comprender cómo pensamos acerca de los robots. Los resultados llevaron al equipo a formular la hipótesis de que los robots pueden pertenecer a una categoría ontológica completamente nueva. Los sujetos no tienden a pensar en robots personificados como vivos, pero tampoco los consideran objetos. Más aún, en los sujetos de tales estudios hubo tendencia a atribuir estados mentales a los robots y les fue difícil participar de determinados comportamientos, como provocarles incomodidad, lo que hubiera sido fácil de haberse tratado de un simple objeto.

Por su parte, Turner (2019: 16) emplea el término robot para referirse a una entidad física o sistema que usa inteligencia artificial. Precisa que, si bien la palabra se usa con frecuencia para describir cualquier tipo de automatización de un proceso por parte de una máquina, agrega un requisito adicional: que la acción la lleve a cabo una entidad que utiliza inteligencia artificial.

En este sentido, compartimos también de manera parcial la propuesta de Turner, en el entendido de que no todos los robots pueden ser considerados «entidades físicas» en el sentido más estricto del término. Sin embargo, consideramos que esta afirmación podría revestir especial importancia para efectos de la propensión al antropomorfismo al que hace referencia Darling (2016: 213-231) y otros autores, como ya señalamos.

En el campo de la robótica, la autonomía se referiría a la posibilidad de adoptar decisiones por uno mismo, sin dejarse influenciar por los demás. En la previsión del Parlamento Europeo, es la capacidad de ser responsable del propio comportamiento y de conducir la existencia, de acuerdo con dictados propios (Ercilla, 2018: 17).

De otro lado, señala Peter Singer que la autonomía es condición necesaria para afirmar que un individuo es un agente moral, teniendo en cuenta que por autonomía se entiende «la capacidad de elegir, de hacer y actuar según las propias decisiones» (Singer, 2011: 84).

Llegado este punto, creemos que debido a la evolución de la robótica $-\mathrm{y}$ en consideración a los avances predecibles en torno a los robots con relación a su estructura, software, conectividad, autonomía, control, entre otros-, optar por un consenso único sobre su definición no sólo es arriesgado, sino que hasta podría llegar a ser limitante dada la propia naturaleza de esta tecnología, por lo que nos alineamos con lo señalado por Jordan. Sin embargo, a efectos del presente trabajo, y a pesar de las discrepancias que pudieran surgir en torno a nuestro planteamiento, consideramos - por ahora - que un robot es un sistema tecnológico altamente evolucionado, complejo y multifuncional, susceptible de presentar diversas tipologías y niveles de desarrollo acorde a los cuales puede «actuar»e «interactuar» en diferentes entornos; al mismo tiempo que, en atención a la estructura «física» que ostente, los despliegues 
serán disímiles. De ahí que es posible identificar sistemas en los que esta «materialidad» o cierta forma de «corporeidad» es evidente, y que son capaces de hacer desplazamiento físico y manipulación del entorno en múltiples niveles de capacidad y en diversos grados; comportamientos ambos que se asocian con mayor frecuencia al término en cuestión, tal vez porque su inserción en el mundo se hizo dentro de esa concepción. Encontramos, de otro lado, sistemas que carecen de esta estructura física, ya que su naturaleza es exclusivamente digital, determinada por un software que permite sus despliegues dentro de la red. Estos agentes artificiales, al ser inteligentes, pueden generar contenidos, consumirlos, influir o alterar de diversas formas nuestro entorno humano y nuestro comportamiento a través de múltiples interacciones de variados contenidos. En la medida en que los sistemas congregan ambas características, se perfilan como una tecnología prometedora de infinitas capacidades y posibilidades, no siempre previsibles. En este orden de ideas, si bien la mayoría de los estudios han abordado los «robots sociales», vinculándolos a una existencia material, nada impediría en el estado actual de las cosas abordar la existencia de los «agentes artificiales sociales». Más aún cuando nuestros despliegues nos alejan cada vez más del mundo físico, en todo orden de cosas.

De otro lado, la inteligencia artificial tampoco ha sido ajena a imprecisiones en cuanto a su conceptualización. La mayoría de las definiciones universales que se han sugerido hasta la fecha se clasifican en una de estas dos categorías: centradas en el hombre y racionalistas. Sobre la primera clasificación, cabe mencionar que el inventor del término inteligencia artificial, John McCarthy, ${ }^{2}$ dijo que todavía no existe «una definición sólida de inteligencia que no dependa de relacionarla con la inteligencia humana». Señala Turner (2019: 12) que muchos enfoques centrados en el ser humano suelen ser en exceso inclusivos e insuficientes. En tanto, en la segunda categoría encontramos un grupo de definiciones más recientes que evitan el vínculo con la humanidad al centrarse en pensar o actuar de manera racional, en el entendido de que este pensar de manera racional significa que un sistema de inteligencia artificial tiene objetivos y razones para alcanzarlos; mientras que actuar de manera racional implica que los sistemas de inteligencia artificial se desempeñen de una manera que pueda describirse como dirigida hacia el objetivo. En este sentido, Nils J. Nilsson (2010: 13) considera que la inteligencia es «esa cualidad que permite a una entidad funcionar adecuadamente y con visión de futuro en su entorno».

Al mismo tiempo, si nos centramos en las clasificaciones que suele recibir la inteligencia artificial, es decir, la estrecha o denominada débil y la general o denominada fuerte, encontramos que las definiciones racionalistas a menudo se basan, ya sea de forma implícita o explícita, en la existencia de objetivos externos para la inteligencia

2. John McCarthy, «Basic questions», What is Artificial Intelligence, noviembre de 2007, disponible en https://stanford.io/36WagmX. 
artificial, por lo que —en opinión de Turner- la dificultad que puede surgir al aplicar tales definiciones a una inteligencia artificial más avanzada y general es que es poco probable que tenga objetivos estáticos por los cuales se pueda evaluar su comportamiento o sus procesos computacionales; más aún, la existencia de objetivos estáticos se convertiría en una condena a la idea de la inteligencia artificial universal.

Refiere también Turner (2019: 16) que la inteligencia artificial es la habilidad de una entidad «no natural» para hacer elecciones por un proceso evaluativo. Precisa que en cuanto a la parte «artificial», es preferible emplear el término no natural a «hecho por el hombre», debido a la propensión de la inteligencia artificial a diseñar y crear otras inteligencias artificiales. Esta es una de sus características emergentes, lo que significa que requiere un tratamiento legal novedoso, ya que la cadena de causalidad entre la inteligencia artificial y su «creador» humano original podría ya no mantenerse. Señala además que la palabra artificial es relativamente incontrovertida, ya que significa algo sintético y que no se da en la naturaleza; la dificultad clave está en la palabra inteligencia, ya que puede describir un rango de atributos o habilidades.

Al mismo tiempo, está implícita en la referencia dada por el autor la toma de decisiones, y que tales decisiones sean autónomas, es decir, que exista un autogobierno (self-governing). En este orden de ideas, señala que la autonomía es diferente de la automatización, en el que un proceso es repetido por una máquina. La autonomía no requiere que la inteligencia artificial instigue su propio funcionamiento: puede tomar una decisión autónoma incluso si ha interactuado con un humano al tomar esa decisión.

Lejos del establecimiento de una eventual definición universal de inteligencia artificial, compartimos parcialmente la opinión de Turner cuando se cuestiona cuál es el factor único de ésta que necesita regulación, debido a que por el momento consideramos que tal vez no exista un único factor, sino varios, y su interrelación conllevaría a múltiples cuestionamientos y planteamientos. Creemos por ahora que la propuesta de Turner en su intento de definición, al señalar que «la inteligencia artificial es la capacidad de una entidad no natural para tomar decisiones mediante un proceso evaluativo», es bastante acertada (Turner, 2019: 16).

En este contexto, Kaplan (2017: 1) señala que la pregunta «¿qué es inteligencia artificial?» es fácil de hacer y difícil de responder. Refiere además que hay muchas definiciones propuestas y cada una con su propio enfoque, pero la mayoría se encuentra más o menos situada en torno a la idea de crear programas de ordenador o máquinas capaces de conductas que consideraríamos inteligentes si las efectuaran seres humanos.

Russell y Norvig (2004: 19), de otro lado, definen a la inteligencia artificial como el estudio de los agentes que reciben percepciones del entorno y llevan a cabo las acciones, señalando que cada agente implementa una función, la cual estructura las secuencias de las percepciones en acciones. 
Boden (2016: 7) señala que parte de la inteligencia artificial fue prevista en la década de 1840 por lady Ada Lovelace, quien se centró en los símbolos y la lógica, sin vislumbrar las redes neuronales, ni la inteligencia artificial evolutiva y dinámica; tampoco tenía ninguna inclinación hacia el objetivo sicológico de ésta, sino que su interés fue puramente tecnológico. Refiere la autora que Lovelace previó que una máquina "podría componer piezas musicales elaboradas y científicas de cualquier grado de complejidad o extensión» y que también podría expresar «los grandes hechos del mundo natural» (Boden, 2016: 7).

Consideramos que la incertidumbre en cuanto a la definición de inteligencia artificial no es la única razón por la que algunos creen que no merece un tratamiento legal separado. En mayor medida, sus usos se extienden a todas las actividades humanas, como ayuda o incluso como un reemplazo para el juicio humano y la toma de decisiones. Pueden ir desde lo inmaterial, como la selección de qué canción tocar a continuación, hasta lo altamente consecuente. En este contexto, las industrias creativas están aprovechando las potencialidades de la inteligencia artificial, y los programas de composición musical fueron algunos de los primeros ejemplos de este desarrollo. ${ }^{3}$

Es innegable que no sólo en términos de capacidad la inteligencia artificial mejora en forma constante, sino también en la medida que su despliegue se incrementa para ser utilizada por personas no expertas. Por ello, consideramos más que probable que la participación humana disminuya con el incremento de su grado de evolución. Cuanto más remoto sea el proceso real de toma de decisiones del diseñador original, más claro será que la inteligencia artificial está haciendo elecciones por sí misma.

\section{Viabilidad de una personalidad jurídica y titularidad de derechos de los robots}

Otra de las preguntas que sin duda genera una infinidad de cuestiones controversiales, al tiempo que por obligación implica el replanteamiento de las instituciones jurídicas tradicionales, gira en torno a la posibilidad de otorgar una personalidad jurídica a los robots, sobre todo si consideramos que existen posiciones diametralmente opuestas sobre el tema: mientras un sector se muestra a favor de planteamientos de diversa índole, otro se encuentra en franca oposición.

Como acertadamente señala Turner (2019: 9), el argumento sobre otorgar a los

3. Jukedeck es una de un número creciente de compañías que utilizan inteligencia artificial para componer música. Sus computadoras aprovechan herramientas como redes neuronales artificiales, modeladas en el cerebro, que permiten que las máquinas aprendan haciendo, como un niño. Tener máquinas para escribir música no es nuevo. En la década de 1950, el compositor Lejaren Hiller usó una computadora para producir la suite Illiac para cuarteto de cuerdas, la primera partitura generada por computadora. Alex Marshall, «From jingles to pop hits, A.I. is music to some ears», The New York Times, 22 de enero de 2017, disponible en https://nyti.ms/36ZxPeK. 
robots personalidad jurídica, al igual como se hace con las empresas, tiene una atracción lógica real. Una empresa no puede actuar de manera independiente, sólo a través de los humanos. En contraste, la inteligencia artificial, aunque formada por humanos -no obstante, quizás solo de manera indirecta- podrá actuar por iniciativa propia. Pero el hecho de que las empresas sólo puedan actuar a través de los seres humanos hace que la noción de que tengan personalidad jurídica y responsabilidad sea menos amenazadora para nuestra idea de normalidad. En ese sentido, el autor considera que dotar de personalidad jurídica a los robots nos hace ver que, al menos en algunos aspectos importantes, son en realidad como personas artificiales.

En esta lógica de pensamiento, el autor aborda la cuestión de la responsabilidad ante un probable daño causado por la inteligencia artificial, así como la titularidad de la propiedad intelectual creada por ésta, al señalar que, ante un eventual otorgamiento de personalidad jurídica, en ambas situaciones la respuesta es simple, mientras que en el caso contrario el escenario se torna espinoso. Como bien afirma Turner, las respuestas podrían centrarse en el creador o proveedor de la inteligencia artificial, o en el caso de que ésta no tuviere un mantenimiento adecuado o presentara alteración de algún tipo, en su operador (si existiese tal persona).

Por consiguiente, y retomando a Savigny cuando considera que las personas morales son el resultado de un artificio legal que la ley elabora por razones de conveniencia - recurriendo entonces a la ficción-, compartimos la opinión de Ercilla (2018: 17) al precisar que devendría en una cuestión de conveniencia la creación de una personalidad jurídica específica para los robots, en consideración a que en un lapso de tiempo medianamente corto la sociedad contará con entes no humanos dotados de voluntad que harán actos susceptibles de crear derechos u obligaciones en el ámbito jurídico.

En este orden de ideas, señala el autor que el panorama del sistema jurídico se conformaría por el reconocimiento de tres personalidades jurídicas: la del ser humano individual dotado de voluntad propia; la de las colectividades humanas, dotadas de voluntad común producto de voluntades aisladas; y la de los sistemas ciberfísicos, dotados de una voluntad surgida de procesos algorítmicos. Sostiene Ercilla (2018: 34) también que, en virtud de la teoría pura del derecho, la persona jurídica, tanto individual como colectiva o electrónica, ${ }^{4}$ no sería sino un conjunto de obligaciones jurídicas y derechos subjetivos, cuya unidad se expresa metafóricamente en el concepto de persona, mientras que el concepto de portador de derechos y obligaciones jurídicas desempeña un papel decisivo en la teoría tradicional de la personalidad jurídica.

Si bien determinar el contenido concreto de los derechos y obligaciones que esta «persona» haya de «portar» deviene en un tema crucial, complejo y altamente deba-

4. Informe con recomendaciones destinadas a la Comisión sobre normas de Derecho civil sobre Robótica, 2015/2103(INL), 27 de enero del 2017, disponible en https://bit.ly/2IIcIDI. 
tible, al no ser objeto de la presente investigación, no será abordado a profundidad y en toda su extensión. Sin embargo, señalaremos algunas ideas que merecen ser objeto de reflexión.

En este contexto, destacamos la importancia del establecimiento de una personalidad jurídica propia y la consecuente denominación de persona por la que se optará, bien sea persona electrofísica, electrónica, robótica, artificial, sintética, una combinación de éstas o - por qué no-persona artificial antropomórfica, denominación que tentativamente postulamos en consideración a la importancia - que por ahora y acorde al estado de las cosas- reviste el factor antropomórfico, ${ }^{5}$ importancia que ha sido plenamente demostrada en los diversos estudios de reconocidos investigadores en el tema, algunos de los cuales han sido considerados a lo largo del presente trabajo, como Balkin, Calo o Darling; al tiempo que es un factor de reiterada identificación en la amplitud de investigaciones científicas que han abordado el tema, debido a sus múltiples implicancias y el impacto implícito señalado por Turkle (2005) o Weinzenbaum (1987).

Sin embargo, probablemente esto conllevaría en su momento a optar también por la búsqueda de una denominación específica para aquellos sistemas que prescinden del factor antropomórfico. De ahí que el abordar lo que denominados por ahora «agentes artificiales inteligentes» implicará múltiples y diversos estudios tendientes a lograr una aproximación al consenso en torno a estos sistemas; al tiempo que nos conducirá, entre otras cosas, a abordar el tema de la singularidad — que no es materia del presente estudio-y retomar el cuestionamiento al antropomorfismo y sus implicancias, pasando por el análisis del concepto de persona en sus múltiples acepciones y en todos sus contextos, ya sea legal, cultural, social, temporal, entre otros.

En este contexto, señala Balkin (2015: 45) que no distingue con claridad entre los robots y los entes con inteligencia artificial, y que a medida que avanza la innovación, la distinción de estos dos tipos de tecnología pueden ser mucho menos importantes para la ley de lo que parece en la actualidad. Agrega que aún no sabe si los límites entre ambas se desdibujarán o diferenciarán cada vez más, de lo que se evidencia que el panorama se vislumbra poco claro aún entre los más entendidos en el tema.

Creemos que al margen de la denominación por la que se opte en cualquiera de los casos, el determinar la naturaleza de «agente moral» que ostentaría esta persona o agente será también un aspecto fundamental, ya que difícilmente se podrá hablar

5. Darling (2016) refiere que las personas somos propensas al antropomorfismo; es decir, proyectamos nuestras propias cualidades inherentes en otras entidades para hacerlas parecer más humanas. Una característica clave de los robots sociales es que están diseñados específicamente para proyectar esas proyecciones. Por su parte, Breazeal (2004) señala que un robot sociable es capaz de comunicarse, interactuar, comprender y aun relacionarse con nosotros de una forma personal. Nosotros, a cambio, debemos poder entenderlo en los mismos términos, relacionarnos y ser empáticos con él. 
de derechos u obligaciones — de diferente índole - atribuibles o exigibles, sin la previa existencia reconocida, sea de la naturaleza que fuere. No obstante, quizá entre muchos cuestionamientos, los más controvertidos al abordar aspectos relacionados a la ética de los robots giran en torno a su capacidad para poder ser portadores de derechos y en virtud de qué criterios serán merecedores de estos. Gunkel (2018: 57) defiende la opinión de que puede haber robots que merecen el estatus legal y moral de tener derechos y desafía los estándares actuales de evaluar qué entidades (corporaciones, animales, etcétera) merecen una posición moral. Refiere que nuestras teorías y prácticas morales se desarrollan y evolucionan desafiando excepciones y limitaciones, y que la ética avanza cuestionando de manera crítica su propia exclusividad hasta eventualmente acomodar a muchas otras antes excluidas o marginadas: mujeres, personas de color, animales, el medio ambiente, entre otras. Cada vez que hay un movimiento para conferir derechos sobre una nueva «entidad», la propuesta está destinada a sonar extraña, aterradora o risible. En este orden de ideas, encontramos detractores como Christine Tiefensee y Johannes Marx (2015: 83), quienes refieren con precisión que los robots no son más que máquinas o herramientas diseñadas para cumplir una función específica. Estas máquinas no tienen intereses ni deseos; no toman decisiones ni persiguen planes de vida; no interpretan, interactúan y aprenden sobre el mundo. En lugar de comprometerse en la toma de decisiones autónomas sobre la base de los objetivos e interpretaciones de su propio entorno, lo único que hacen es ejecutar un programa preinstalado. En resumen, los autores consideran que los robots son autómatas inanimados, no agentes autónomos. Como tales, ni siquiera son el tipo de objeto que podría tener un estatus moral.

En este sentido señala Balkin (2015: 45) que Calo - de manera acertada-sugiere que todavía estamos muy lejos de tratar a los robots y agentes de inteligencia artificial como entidades conscientes de derechos o responsabilidad. Agrega que al referirse a los robots incluye también a los agentes de inteligencia artificial y algoritmos de aprendizaje automático, y que las personas parecen temer a todos. Considera que estamos pasando a una sociedad organizada en torno a la toma de decisiones sociales y económicas mediante algoritmos, robots y agentes de inteligencia artificial, los cuales no sólo toman las decisiones, sino que en algunos casos las ejecutan.

Gunkel (2018: 12) por su parte refiere que los términos robot y derechos ya son lo suficientemente complicados, pero una vez que se reúnen las dos palabras, se obtiene una reacción alérgica. El autor planteó y analizó la siguiente pregunta: «¿Pueden y deben los robots tener derechos?». Es evidente que este cuestionamiento se compone de dos aspectos separados y diferentes: «¿Pueden los robots tener derechos?», que es una pregunta que se refiere a las capacidades ontológicas de una entidad en particular; $\mathrm{y}$ «ideben los robots tener derechos?», que es una pregunta que indaga sobre las obligaciones normativas frente a esta entidad. Estas dos preguntas invocan y opera- 
cionalizan una distinción conceptual bastante famosa en filosofía, conocida como «el problema del ser y el deber ser» $\mathrm{o}$ «la guillotina de Hume». ${ }^{6}$

En consecuencia, el debate ético - en especial cuando se trata de los derechos de los demás - suele estar basado en diferentes supuestos ontológicos de hecho, que luego se toman como la base racional del valor moral y la toma de decisiones.

Para muchos, los «derechos de los robots» son simplemente impensables, lo que significa que es imposible pensar en la medida en que el concepto mismo se opone al sentido común o al buen razonamiento científico; o debe evitarse a propósito, como algo que no debe pensarse, es decir, como una especie de idea prohibida que abriría una caja de Pandora y por lo tanto debe ser reprimida. Cualquiera fuese la razón, existe una decisión deliberada y un esfuerzo concertado para no pensar o al menos no tomar como un asunto serio para pensar la cuestión de los derechos de los robots.

Sin embargo, considera el autor que ésta es precisamente la razón por la que debemos pensar lo impensable y desafiar estas declaraciones, suposiciones u ortodoxias, y esto tiene que suceder por al menos dos razones. En primer lugar, cualquier declaración dogmática de este tipo ya debería inquietarnos y hacernos sospechar. El hecho de que este pensamiento - el sólo pensamiento que surge en la asociación de las dos palabras robot y derechos - haya sido declarado impensable debería hacernos dar una pausa conducente a plantearnos una serie de preguntas críticas, como: ¿quién dice? ¿Quién decide de antemano lo que podemos o no podemos pensar? Y, quizás más revelador aún: ¿qué valores y supuestos se protegen a través de este tipo de proscripción y prohibición?

A estas preguntas nos atrevemos a sumar otras: ¿qué intereses se encuentran de por medio? y ¿a quienes pertenecen los intereses que se pretenden resguardar? No creemos que la respuesta única sea al ser humano de manera absoluta y general. Sólo por citar uno de estos intereses, bastaría analizar la diversidad de planteamientos que se están dando en el ámbito de la tributación en torno al tema.

En segundo lugar, desafiar las exclusiones y prohibiciones es el trabajo apropiado de la ética, que opera tomando decisiones exclusivas, inevitablemente elige ganadores y perdedores, y determina quién o qué está dentro de la comunidad moral y quién o qué permanece fuera, o en la periferia.

A diferencia del término robot, el término derechos tiene una caracterización bastante estricta y formal. Para la mayoría de los filósofos y juristas morales, como sugiere Wenar, ${ }^{7}$ los incidentes hohfeldianos (privilegios, reclamos, poderes e inmunidades, ya sean considerados de forma aislada o en alguna combinación) proporcionan lo

6. Marshall, «From jingles...».

7. Leif Wenar, The Stanford encyclopedia of philosophy, s. v. «rights». Disponible en https://stanford. io/2FnQMfR. 
que se considera una formulación completa y explican lo que entendemos por el término derechos.

La complicación surge en el momento de determinar quién o qué puede o debería tener una participación en estos cuatro incidentes. En otras palabras ¿quién o qué puede tener derechos?

Existen dos teorías que compiten para abordar esta cuestión: la teoría de la voluntad y la teoría del interés. La primera establece una barrera bastante alta, que requiere que el sujeto de un derecho posea la autoridad o la capacidad de afirmar por sí solo un privilegio, reclamo, poder o inmunidad que deberían ser respetados por otros. La segunda, en cambio, proporciona un umbral mucho más bajo, lo que hace posible que uno pueda decidir los derechos en nombre de los intereses de los demás.

Cabe señalar que algunos países asiáticos ya han abordado la regulación de los robots dentro de sus ordenamientos jurídicos y no exclusivamente dentro de un marco de estandarización de patrones industriales, sino centrándola en la interacción humana y el impacto social. En este sentido, proponemos como primer derecho de los robots sociales: el derecho a la integridad y a no sufrir daño - lo que fácilmente podría ser extensible y aplicable a otros tipos de sistemas previas consideraciones en torno su naturaleza-, derecho que sería inalienable y guardaría congruencia con nuestra tendencia a proteger aquello con lo que nos relacionamos de manera permanente y cercana, o con aquello hacia lo cual desarrollamos ciertos lazos que denotan grados de intensidad. Consideramos además que en cierto punto no podremos negar que este derecho probablemente oculte la preocupación por proteger más nuestro propio estado emocional que otro criterio biológico objetivo. En consecuencia, reviste un carácter discutible, al igual que todas las temáticas que pretendan abordar el binomio robótica e inteligencia artificial.

Pagallo (2013: 21), a la hora de abordar la sustantividad de un derecho de los robots, pone de relieve cómo las tendencias en los derechos comparados pueden sistematizarse en tres grandes respuestas, que van desde la afectación de las concepciones tradicionales hasta una negación sobre su especificidad. Por su parte, señala Balkin (2015: 57) que Calo afirma bien cuando habla de «una nueva categoría de sujeto legal a medio camino entre la persona y el objeto». La ubicación es «a medio camino» porque la asignación de estado puede ser incompleta, contextual, inestable y, sobre todo, oportunista. Las personas pueden tratar al robot como una persona $-\mathrm{o}$ animalpara algunos propósitos y como un objeto para otros.

Esta nueva personalidad jurídica encontrará asidero en la medida que se reconozca y acepte que no estaremos frente a entes sometidos a la total voluntad humana, sino que también serán consecuencia del entorno que los rodea, lo cual le permitirá adoptar decisiones en virtud de aprendizajes autodidactas y realidades circundantes. De ahí que las implicancias en todo orden de cosas - que acompañarán a esta nueva 
realidad en la que estamos cada vez más inmersos - avizoran un panorama que fue sólo concebido por la ciencia ficción.

Llegado este punto, cabe señalar que evidenciamos - al menos en el contextodos existencias robóticas, ambas identificables y diferenciadas en cierta medida. Una de ellas encuentra en el elemento antropomórfico, un factor esencial e indispensable dentro de la dupla robótica e inteligencia artificial. La otra pareciera aún no contar con un bagaje de investigación tan profundo en el sentido que nos ocupa, es decir, dentro de la esfera legal; más aún, algunos autores excluyen expresamente los sistemas en los cuales la inteligencia artificial no se encuentra vinculada a un tipo determinado de embodied, como señalara Winfield (2012: 22), que le permita "ser», según Calo (2015: 531). Luego este autor asume una postura susceptible de diversos cuestionamientos al referirse a la ciudadanía de los robots. ${ }^{8}$

Siguiendo esta línea de pensamiento, y si nos centramos en el factor antropomórfico, somos de la opinión de que el primer paso para poder establecer un verdadero derecho de los androides - postulado que por ahora consideramos más preciso frente al genérico «derecho de los robots» y más pertinente según los planteamientos y enfoques abordados en el presente trabajo- es la elaboración de códigos de conducta ética que sean de doble observancia, tanto por los ingenieros en el transcurso de sus investigaciones y el proceso de diseño y fabricación, como en el transcurso de la programación específica que se les dará a los sistemas. Sin embargo, consideramos que, en relación con las diferentes existencias, llegado el momento será necesario establecer - entre otros - características inherentes y elementos determinantes con miras al establecimiento de una adecuada tutela, sin incurrir en discriminaciones o exclusiones de algún tipo y en el entendido de que diversas formas de sistemas dotados de inteligencia artificial estarán cumpliendo o desarrollando actividades de la misma naturaleza, como podría ser la creación de diversos tipos de obras.

\section{Los robots en las creaciones artísticas: Arte en sí mismos o creadores de arte}

Boden (2011: 12) precisa que la distinción entre art y craft conlleva una gran acumulación de bagaje intelectual y una larga historia de controversia filosófica. También tienen, al menos en nuestra cultura, una serie de diferencias sociológicas. Refiere la autora que los practicantes de art y craft tienden a pertenecer a distintos grupos profesionales, y aborda los diversos criterios controvertidos de carácter distintivo que evidencian varios tipos de esnobismo, rivalidad y actitud defensiva en la atribución de estos términos.

8. Ryan Calo, «Much ado about robots», Cato Unbound, 11 de abril de 2018, disponible en https://bit. ly/3dmUhj8. 
Es importante hacer referencia a ellos, porque nos permiten abordar el hecho de que no existe una sola noción de lo que es el arte como tal o de lo que en sentido estricto - según determinadas consideraciones- podría abarcar. Esto hace posible reflexionar en torno a la existencia de aquellas características mínimas inherentes sobre una obra que la harían ostentar el calificativo de tal —entendiéndola en sentido amplio-, ya que muchas veces los criterios establecidos incurriendo en la esfera de la subjetividad se convertirían en límites demarcatorios excluyentes.

En la medida que la tecnología ha ido cobrando un rol cada más trascendente y generalizado en la cultura actual, ha ido surgiendo la imperiosa necesidad de analizar las consideraciones en torno al papel que ha desempeñado la robótica en el arte contemporáneo; evolución que ha sido contemplada bajo diferentes denominaciones. En opinión de Eduardo Kac (2001: 77), muchos trabajos destacados en este campo a menudo evaden cualquier definición limitada de robótica, con excepción, tal vez, del principio de prioridad del comportamiento sobre la forma. Sin embargo, precisa el autor que apegarse a una definición estrecha parece menos importante que la oportunidad de trazar paralelos entre las estrategias que a veces ponen en primer plano a las criaturas electrónicas, situación a la que Kac ha denominado «arte robótico», a la combinación de lo orgánico y electrónico lo ha considerado como «arte cibernético» y a la proyección remota de un sujeto humano sobre un telerobot, «arte de telepresencia». Precisa además que estas formas de arte no sólo parecen relacionadas conceptualmente de manera directa, sino que también aparecen hibridadas en diversas obras.

Kac (2001: 76) señala que una de las cuestiones más problemáticas de la robótica en el arte es la definición misma de lo que es un robot. Sin embargo, otro aspecto relacionado es la misma definición operativa de los robots que se encuentran tanto en la investigación científica como en las aplicaciones industriales, ya que, desde esta última perspectiva, los robots son dispositivos electromecánicos avanzados controlados por computadora. El autor considera que, a medida que los artistas han empujado los límites del arte, introdujeron la robótica como un nuevo medio y desafiaron nuestra comprensión de los robots, cuestionando, por lo tanto, nuestras premisas para concebir, construir y emplear estas criaturas electrónicas A esto se suma el hecho de que la inteligencia artificial ha estado generando resultados creativos durante décadas y ahora el crecimiento continuo y exponencial de la potencia informática está conduciendo a las máquinas creativas a convertirse en los principales impulsores del crecimiento económico.

Desde que hicieron su aparición en el campo de las artes, los robots cautivaron a las sociedades de todos los tiempos. En ese contexto, refieren Elizabeth Stephens y Tara Heffernan (2016: 29) que la Dama Musical, una autómata del siglo XVIII que actualmente se exhibe en el Musée d'Art et d'Histoire en Neuchâtel (Suiza), fue considerada como uno de los primeros robots programables del mundo, y catalogada 
durante su exposición en Londres en el año de 1776 como una maravilla tecnológica. Era un robot consistente en una figura que se movía por medios mecánicos y tenía apariencia inteligente, capaz de participar en la producción de una forma de arte humano como es la música.

A lo largo de la década de 1700, una serie de figuras mecánicas, inquietantes y realistas cautivaron al público de ese entonces, al ejecutar hazañas que denotaban habilidad e inteligencia. Entre estos, se encontraba el Flautista de Jacques Vaucanson, una elaborada computadora musical humanoide que se dio a conocer en 1738 y a la cual seguirían otras creaciones de su misma naturaleza. Estos autómatas, al igual que otras figuras mecánicas que fascinaron en el siglo XVIII, no fueron solo productos de arte y habilidad técnica, sino también productores altamente calificados del arte en sí mismo y, en consecuencia, partícipes de actividades culturales que fueron consideradas siempre de exclusivo dominio humano.

En opinión de Riskin (2003: 612), la atención que congregaron en su momento se debió a que dramatizaron al mismo tiempo dos afirmaciones contradictorias: que las criaturas vivientes eran esencialmente máquinas y que las criaturas vivientes eran la antítesis de las máquinas. En este contexto, como era de esperarse, empezaron a surgir los detractores dentro de este campo: Wood (2007:17) señaló que el intento de crear vida mecánica era algo preocupante en estas figuras automatizadas y argumentó que eran evidencia del peligro creciente del racionalismo y la ambición científica.

Mientras que en el siglo XVIII fue la apariencia humana del autómata lo que fascinó a los artistas e ingenieros, a lo largo del siglo XIX, a medida que la era de la industrialización continuó transformando el paisaje cultural y el escenario físico, la producción y exhibición de los autómatas no se detuvo. Sin embargo, su rol en el imaginario cultural inició su transformación debido al uso e importancia que empezó a cobrar la mecánica. En este orden de ideas, la invención de la palabra robot en sí misma se constituyó en una muestra representativa de este cambio de época. La invención de Čapek de la idea contemporánea del robot fue una respuesta directa a las radicales transformaciones culturales y económicas generadas por la creciente industrialización; de ahí que resulta comprensible que sus robots fueran el producto de un imaginario maquinista del siglo XIX o el resultado de una era de producción industrial en masa.

En el siglo XX fueron los procesos y funciones de la tecnología los que recibieron la mayor atención. A lo largo de este siglo, la experimentación con la robótica produjo nuevos campos en las artes, definidos por sus compromisos interdisciplinarios con tecnologías mediante las cuales la producción de arte podría automatizarse de varias maneras. Al mismo tiempo, la experimentación artística de los siglos XX y XXI con la robótica marcaron un cambio significativo frente a su historia temprana.

Kac (2005: 170) considera que el problema teórico central de la era del arte digital ha sido la oposición radical entre la cultura de la informática y la cultura de las artes. 
De ahí que el arte robótico es importante no sólo porque se convierte en un punto de apoyo entre la abstracción de la informática y la materialidad situada del arte, sino también porque es crucial en el desarrollo de las prácticas artísticas digitales debido a que la robótica implica el diseño de modalidades de interacción y la necesidad de una teorización de ésta, al tiempo que abarca ese campo en una extensión más amplia: la estética del comportamiento.

El autor (Kac, 2001: 78), citando a Paik, Shannon e Ihnatowicz, señala que el control remoto, las entidades cibernéticas y el comportamiento autónomo, como lo describieron por primera vez estos tres autores, han definido las tres direcciones clave que han instruido el desarrollo de la robótica en el arte. Precisan que debido a que en la actualidad la libertad artística promueve la diversidad robótica, la comprensión de este marco triangular es esencial para permitirnos continuar explorando la historia, la teoría y la creación del arte robótico.

Por su parte, Simon Penny (2016: 49) refiere que la robótica, como campo, se caracteriza por dos cualidades: primero, implica el diseño del comportamiento; y segundo, cierra la brecha entre el mundo inmaterial de la informática y el código y las exigencias de la materialidad. Estas dos cualidades definitorias dejan entrever la existencia de un conjunto importante de relaciones con el arte en su tradicional entendido.

Somos de la opinión de que el arte robótico implica una interacción con el espectador, ya sea de manera activa o de forma pasiva; al tiempo que las creaciones de arte más grandiosas y abrumadoras siguen siendo acertijos sin resolver, aun sin involucrar a la tecnología.

\section{Entre la creatividad computacional y la creatividad humana}

El campo de las artes no ha sido ajeno a la influencia del desarrollo tecnológico. Con el tiempo, los robots pasaron de ser considerados obras de arte en sí mismos a participar en la producción de alguna forma de arte y en la actualidad, independientemente de su naturaleza, a ser considerados por algunos sectores como creadores de arte.

Margaret Boden (2015:5) conceptualiza a la creatividad computacional como el uso de computadoras para generar resultados que se considerarían creativos si fueran producidos sólo por humanos. Refiere además qué, estrictamente hablando, esto incluye no sólo arte, sino también teorías científicas innovadoras, conceptos matemáticos y diseños de ingeniería. Pero el término se usa a menudo aplicado sobre todo a resultados que tienen interés artístico. Señala que a principios de la década de 1960, los artistas de vanguardia del Institut de Recherche et Coordination Acoustique/Musique (IRCAM) en París comenzaron a experimentar con la música por computadora, aunque se centraron más en la instrumentación que en la composición. En 1968, la reunión de la International Federation for Information Processing (IFIP) en Edim- 
burgo incluyó un concurso de música compuesta por computadora, que condujo a la fundación de la Computer Arts Society (CAS).

Algunos ejemplos de arte de creatividad computacional muy temprano se exhibieron de manera individual a mediados de la década de 1960. El primero, en 1963, fueron los dibujos de Joan Shogren generados por computadora y presentados en la tienda de libros Spartan en San José, California. Otros trabajos que recibieron más atención fueron los de Georg Nees, Michael Noll y Frieder Nake, que mostraron en Stuttgart y Nueva York en 1965. Eran diseños muy abstractos, casi geométricos, generados por programas escritos no por artistas profesionales, sino por matemáticos.

En esta misma época, Harold Cohen en el campo de artes gráficas y David Cope dentro de la música, se embarcaron en un proyecto que duraría dos décadas, ejerciendo una gran influencia en el arte de la creatividad computacional a principios de 1980; el primero con su programa AARON y el segundo con Emmy, ambos capaces de explorar un espacio dado de manera aceptable; en tanto, existen otros programas que pueden incluso transformar su espacio conceptual, alterando sus propias reglas. A fines de la década de 1980, nuevos tipos de computación estuvieron disponibles, estos incluyeron el conexionismo PDP y la programación evolutiva. John Holland, que definió formalmente el primer algoritmo genético a principios de la década de 1970, había descrito la informática evolutiva hacía más de veinte años, pero su algoritmo no se había implementado debido a la falta de energía de la computadora. En la década de 1990, al existir las máquinas necesarias, ambas nuevas metodologías de inteligencia artificial atrajeron el interés de los artistas de creatividad computacional.

Hoy, la creatividad computacional ha dado lugar a varios géneros de arte por computadora, además de fomentar innumerables estilos individuales. La creatividad computacional se encuentra en una posición difícil, ya que su principal pregunta de investigación se refiere a un concepto en esencia debatible. De acuerdo con ciertos entendimientos, a la pregunta «ipueden las máquinas ser creativas?», se puede responder con una negativa, sin mayor elaboración o debate en torno al tema.

Boden (2011: 29) conceptualiza a la creatividad como la capacidad de generar ideas o artefactos que sean nuevos, sorprendentes y valiosos. Al mismo tiempo, señala que entra en casi todos los aspectos de la vida no es una «facultad» especial, sino un aspecto de la inteligencia humana en general, y no es un asunto de todo o nada. En lugar de preguntar si una idea es o no creativa, deberíamos preguntarnos cuán creativa es y de qué manera.

Debido a que la creatividad, por definición, implica no sólo novedad sino también valor, y debido a que los valores son muy variables, se deduce que muchos argumentos sobre la creatividad se basan en desacuerdos sobre el valor. Esto se aplica a las actividades humanas no menos que al rendimiento de la computadora. Entonces, incluso si pudiéramos identificar y programar nuestros valores estéticos a fin de per- 
mitir que la computadora informe y monitoree sus propias actividades en secuencia, seguiría existiendo desacuerdo sobre si la computadora parece ser creativa.

Boden (2011: 30) hace una distinción entre la creatividad psicológica (P-creatividad) y la creatividad histórica (H-creatividad). La primera implica presentar una idea sorprendente y valiosa que es nueva para la persona que se le ocurre; pero si una nueva idea es H-creativa, eso significa que nadie más la ha tenido antes: ha surgido por primera vez en la historia humana. Precisa la autora que esta es la importante y un caso especial de creatividad $\mathrm{P}$, y que si «nuevo» en este contexto tiene dos significados muy diferentes, «sorprendente» tiene tres.

Así, la creatividad puede suceder de tres formas principales, correspondientes a los tipos de sorpresa. En ese contexto, señala la autora que los tres caminos para sorprender son las tres formas de creatividad: combinacional, exploratoria y transformacional. Indica además que para comprender cómo puede suceder la creatividad exploratoria o transformadora, debemos saber qué son los espacios conceptuales y qué tipo de procesos mentales podrían explorarlos y modificarlos (Boden, 2011).

Señala Boden (2011: 36) que muchas personas argumentarán que ningún computador puede ser genuinamente creativo, sin importar su rendimiento. Incluso si superara de lejos al científico ordinario o al artista callejero, seguirían sin considerarlo creativo, a pesar de producir teorías o música de alto valor. Es que, como bien refiere la autora, existen diferentes argumentos usados comúnmente para sostener esta postura, como que es la creatividad del programador la que trabaja, no la máquina. Esta última no es consciente y no tiene deseos, preferencias o valores, por lo que no puede apreciar ni juzgar lo que está haciendo. Una obra de arte es una expresión de la experiencia humana o una comunicación entre seres humanos, por lo que las máquinas simplemente no cuentan.

Es evidente que, de aceptar esta razón u otras alineadas a esta postura, significaría que no habría nada más que discutir en torno al tema. Sin embargo, Boden precisa que esto no es así, ya que estas objeciones aceptan, en aras de la argumentación, que el rendimiento de un computador imaginario es muy similar al de los seres humanos, sea o no monótono, por lo que de hecho la discusión gira en torno a establecer si es cierto que los computadores podrían presentar ideas que al menos parezcan creativas.

En este contexto, la creatividad combinacional - que implica hacer conexiones desconocidas entre ideas familiares - es fácil de modelar en un computador, porque nada es más simple que elegir dos ideas (dos estructuras de datos) y ponerlas una al lado de la otra. Esto incluso se puede hacer con cierta sutileza, utilizando métodos conexionistas, es decir, una computadora podría producir nuevas combinaciones de manera permanente.

Boden (2016: 1) señala que la inteligencia artificial busca hacer que las computa- 
doras hagan el tipo de cosas que las mentes pueden hacer. La inteligencia no es una dimensión única, sino un espacio ricamente estructurado de diversas capacidades de procesamiento de información. En consecuencia, la inteligencia artificial utiliza muchas técnicas y aborda muchas tareas diferentes.

\section{Perspectivas y tratamientos legislativos: Autoría de la inteligencia artificial}

Aunque el término autor se menciona y utiliza a menudo a lo largo del texto del Convenio de Berna - uno de los tratados internacionales que, junto con el Tratado de la OMPI sobre Derechos de Autor y el Acuerdo sobre los ADPIC, constituyen los documentos más importantes sobre el tema- no se le define en forma explícita. Es probable que la razón de esto radique en que cuando el Convenio y sus posteriores revisiones fueron discutidos, existió un entendimiento similar entre los países miembros respecto de quién era considerado autor, por lo que, en ese momento, mayor interpretación o explicación no fueron necesarias. De otro lado, tanto el Tratado como el Acuerdo guardan silencio respecto de la definición de autor, aunque ambos exigen el cumplimiento del Convenio de Berna. Consideramos que, debido a que estos documentos internacionales dejan a discreción de cada jurisdicción nacional la definición, la diversidad podría generar obstáculos que demandarán algún nivel de armonización, sobre todo respecto de conceptos clave.

En posteriores revisiones, las divergencias entre las leyes nacionales sobre otros aspectos de la autoría se han vuelto más pronunciadas. Una de ellas es el grado real de creación intelectual requerida para cumplir con los criterios de originalidad, entendiéndola en el sentido de «individualidad» y no de novedad en sentido estricto, ya que se exige que el producto creativo, por su forma de expresión, tenga sus propias características para distinguirlo de cualquier otro del mismo género. En todo caso, siguiendo el planteamiento de Satanowsky (1954: 470), quien sostenía que la originalidad se presume y quien la niegue debe probarla, se posibilita el debate en torno a la originalidad producto de un ser humano o de una inteligencia artificial.

Los sistemas de derecho consuetudinario enfatizan tradicionalmente el grado de habilidad y trabajo involucrado, mientras que los países de tradición romano-germánica tienden a poner más peso en el nivel de creatividad. De hecho, este tema también puede referirse de manera indirecta a las preguntas sobre si es necesario que un autor sea una persona física o jurídica.

En este contexto, en 2016 la Organización Mundial de la Propiedad Intelectual (OMPI) precisó que el continuo desarrollo de la tecnología de redes digitales produce información con un valor indefinible, de acuerdo con la noción de creatividad, y agregó que ejemplo de esto serían la música o las imágenes producidas a partir de 
una inteligencia artificial. ${ }^{9}$ Al tiempo que, como parte constitutiva del primer pilar vinculado a innovación, ${ }^{10}$ abordó la construcción de un nuevo sistema de derechos de autor, además de examinar la naturaleza de la protección de la propiedad intelectual para nuevos bienes de información como las obras creadas de forma autónoma por inteligencia artificial. En este sentido, producto de la primera sesión de diálogo sobre inteligencia artificial y propiedad intelectual, ${ }^{11}$ elaboró el borrador de documento temático sobre políticas en torno a estos temas. Su posterior revisión ${ }^{12}$ recogió entre otras cosas la cada vez mayor capacidad de las aplicaciones de inteligencia artificial para generar obras literarias y artísticas; además, a diferencia del primer documento, se obvió «de forma autónoma». En concreto, respecto de la autoría y titularidad de los derechos, se plantearon diversas interrogantes que abordaron la atribución, titularidad y posibilidad de otorgar personalidad jurídica a una aplicación de inteligencia artificial ante la generación autónoma de una obra, así como el establecimiento de un sistema sui generis de protección para estas creaciones. Aún no existe una versión final del documento y una nueva revisión está prevista. En este contexto, cabe señalar que Guadamuz ${ }^{13}$ refiere que nunca se ha prohibido en forma expresa conceder derechos de autor sobre las obras generadas por inteligencia artificial.

Yanisky-Ravid (2017: 664) señala que los sistemas de inteligencia artificial y el aprendizaje automático ya se han convertido en parte de nuestra vida cotidiana, $y$ $\mathrm{n}$ la actualidad se pueden identificar sistemas de inteligencia artificial en áreas en la cuales años atrás jamás se hubiera imaginado. En este contexto, el autor propone un nuevo modelo de responsabilidad para las obras generadas por estos sistemas, llamado modelo work made for hire (WMFH). Este modelo ve el sistema de inteligencia artificial como un empleado creativo o un contratista independiente del usuario. Así, la propiedad, el control y la responsabilidad se impondrían a las personas o entidades legales que usan sistemas de inteligencia artificial y disfrutan de sus beneficios (Yanisky, 2017: 660).

El autor considera que su propuesta refleja y mantiene las características humanas del sistema de inteligencia artificial, como la independencia, la creatividad y la

9. «Intellectual property strategic program 2016», Organización Mundial de la Propiedad Intelectual, mayo de 2016, p. 10, disponible en https://bit.ly/30Ymzor.

10. «Intellectual...», 6 .

11. «WIPO Conversation on intellectual property (IP) and artificial intelligence (AI)», Organización Mundial de la Propiedad Intelectual, WIPO/IP/AI/2/GE/20/1, 13 de diciembre de 2019, disponible en https://bit.ly/2SOgtfE.

12. «WIPO Conversation on intellectual property (IP) and artificial intelligence (AI): Second session», Organización Mundial de la Propiedad Intelectual, WIPO/IP/AI/2/GE/20/1 REV, 21 de mayo de 2020, disponible en https://bit.ly/3718m4x.

13. Andrés Gaudamuz, «La inteligencia artificial y el derecho de autor», OMPI Revista, octubre de 2017, disponible en https://bit.ly/30Zp9Be. 
inteligencia; y que, por otro lado, asegura que el empleador o el usuario mantengan los derechos y deberes apropiados, incluida la responsabilidad por los resultados. Considera que ésta puede ser la mejor solución para el problema actual de la falta de responsabilidad para los sistemas de inteligencia artificial independientes. Más aún, sostiene que ver el sistema de inteligencia artificial a través del lente de derechos de autor proporcionará nuevas oportunidades para imponer la propiedad y la responsabilidad de las entidades legales conocidas y que la implementación de un modelo WMFH modificado puede estructurar una solución factible en el futuro cercano e imponer responsabilidades a los usuarios que tienen afinidad con los sistemas de inteligencia artificial.

Sin embargo, somos de la opinión de que aún en el caso de acogerse esta propuesta, el hecho de considerar a la inteligencia artificial como «un empleado creativo o un contratista independiente del usuario» implicaría necesariamente un previo reconocimiento y concesión de derechos y, por lógica consecuencial, una anterior determinación de personalidad a la cual le sean atribuibles.

De ser así, este punto nos lleva a reflexionar sobre las diversas posturas ya analizadas, los cuestionamientos abordados y las propuestas planteadas, además de considerar las diversas y múltiples posibilidades existentes respecto de esta tecnología disruptiva.

Tradicionalmente, la titularidad del derecho de autor sobre las obras generadas por un computador no era cuestionable, debido a que el programa era tan sólo una herramienta de apoyo al proceso creativo y no era quien tomaba las decisiones asociadas al proceso en sí mismo y sin intervención humana. Sin embargo, el incremento de la creación de obras mediante la inteligencia artificial empieza a generar diversas implicancias no previstas hasta ahora en casi ningún ordenamiento legislativo, con excepción tal vez de China, país donde el Tribunal de Nanshan dictaminó recientemente que un «trabajo» generado por inteligencia artificial califica para la protección de los derechos de autor y disputas de competencia desleal, determinando que Shanghai Yingxun Technology Company infringió los derechos de autor de Tencent y debería asumir responsabilidad civil. ${ }^{14} \mathrm{El}$ tribunal consideró que la forma de expresión del artículo ${ }^{15}$ se ajustaba a los requisitos del trabajo escrito y el contenido mostraba la selección, análisis y juicio de la información y los datos relevantes del

\footnotetext{
14. Sentencia del Tribunal Popular del distrito de Shenzhen Nanshan, 11 de septiembre de 2019, disponible en https://bit.ly/3iVvIeq.

15. Tencent desarrolló en el 2015 un programa automatizado de redacción de noticias basado en datos y algoritmos, que bautizó como Dreamwriter. En agosto de 2018, Dreamwriter escribió un informe financiero que incluía en índice de Shanghai de ese día, las divisas y los flujos de capital. El artículo publicado en el sitio web de Tencent Securities señaló que «el artículo fue escrito automáticamente por Tencent Robot Dreamwriter». Shanghai Yingxun Technology Company luego copió el artículo en su propio sitio web y lo difundió ese mismo día.
} 
mercado de valores. También estableció que su estructura era razonable, la lógica era clara y tenía cierta originalidad.

Como era de esperarse, esta decisión generó diversas posturas en torno al tema. Entre otras cosas, se señaló que, de acuerdo con la ley de derechos de autor y algunas convenciones internacionales, la definición de un trabajo enfatiza primero que la creación sea original, reproducible y producida en base a la actividad intelectual humana. ${ }^{16}$ Por lo tanto, la inteligencia humana es el núcleo y la premisa. Se discutió también que si el contenido fue producido por máquinas después de que las personas escribieran algunas palabras clave, entonces las máquinas deberían ser el autor en lugar de la inteligencia humana y el contenido no debería estar protegido en el sentido de la ley de derechos de autor; cuestionándose que si cualquiera puede usar las máquinas y generar el mismo contenido con las mismas palabras clave, debe replantearse qué es lo que protege exactamente la ley de derechos de autor: la actividad intelectual de elegir palabras clave o un trabajo realmente creado por la inteligencia humana.

En este orden de ideas, cabe señalar que la ley de derechos de autor de china adopta el principio de adquisición automática, es decir, el autor obtiene automáticamente los derechos de autor de la obra después de haberla creado. El registro de derechos de autor no es necesario para la generación de derechos, sino tan sólo como evidencia tendiente a demostrar la existencia de derechos e intereses y para resolver disputas sobre derechos (Zhang, 2019: 513). Sin embargo, en un contexto que avizora la posible existencia masiva de creaciones producidas por la inteligencia artificial, será necesaria la exigencia de registro de los trabajos producidos por ésta a efectos de una confirmación de derechos.

Es indiscutible que con el desarrollo de la inteligencia artificial, esta tecnología se ha utilizado para generar algunas obras tradicionalmente supeditadas a la creatividad humana. Sin embargo, si tales trabajos debieran estar protegidos por el sistema de derechos de autor es un tema aún discutible por muchos sectores y sigue siendo materia de análisis.

El caso en mención nos conduce a reflexionar sobre la posibilidad de generar sistemas alternos de protección que tal vez no necesariamente estarían sujetos a la denominación de derechos de autor, o al menos no como hasta ahora se conocen y de la forma en que se han venido tutelando; pero que, según el estado de la evolución tecnológica, consideramos deviene en una tarea que no puede seguir siendo postergada por mucho más tiempo.

Sin duda, China ha sentado un precedente interesante que más allá de ser o no ser cuestionable o de ver en qué medida se produciría este cuestionamiento, nos lleva

16. Zhang Yangfei, «Court rules AI-written article has copyright», China Daily, 9 de enero de 2020, disponible en https://bit.ly/312ZUOi. 
analizar una protección que puede ir más allá de los derechos intelectuales, como sería la relacionada a la competencia desleal o la posibilidad de abordar la adopción de un sistema tuitivo conjunto, como se produce con algunas figuras clásicas de la propiedad industrial que comparten protección con los derechos de autor.

Refiere Ihalainen (2018: 727) que una de las pocas naciones que avanza en el debate sobre la legislación de la inteligencia artificial en materia de derechos de autor es Japón, cuyo Gobierno ha estado considerando un nuevo régimen de protección para la propiedad intelectual no creada por humanos. Esta nueva legislación buscaría limitar la protección de las obras creadas por inteligencia artificial, tratándolas como si fueran marcas registradas y realineando su protección a la competencia desleal en lugar de un esquema más amplio basado en expresiones. El grado de protección de las obras en particular también estaría vinculado a su popularidad, de manera similar al concepto de buena voluntad, dejando obras menos oscuras o populares con poca o ninguna protección. Las obras serían propiedad de la persona o la empresa que creó la inteligencia artificial en cuestión.

En un artículo publicado por la OMPI, refiere Guadamuz ${ }^{17}$ que hay indicios de que la legislación de numerosos países no es favorable al derecho de autor que no se aplique al ser humano, como España y Alemania, donde únicamente las obras creadas por un ser humano pueden estar protegidas. En el mismo sentido, en Estados Unidos la ley de derechos de autor sólo protege los frutos del trabajo intelectual que se basan en los poderes creativos de la mente, por lo que por el momento no existe protección para las obras creadas por una inteligencia artificial, en tanto que el uso de la tecnología por parte de un humano para crear lo mismo que una inteligencia artificial sí estará protegido. La Oficina de Derechos de Autor de los Estados Unidos ya había destacado esto en 1965 como un problema futuro (Ihalainen, 2018: 726). Sin embargo, parece haber sido ignorado en los años que le siguieron.

En países como España, Colombia, Brasil, México o Australia, el concepto de derecho de autor está ligado a una persona, lo que dificultaría la protección de este nuevo tipo de obras. A su vez, señala Ihalainen (2018: 726) que la legislación australiana no tiene disposiciones para hacer el «arreglo necesario» para la creación de la obra y duda de la existencia de derechos de autor en obras creadas por la inteligencia artificial, incluso con aportes humanos. Se sugirió que esto podría implementarse para viabilizar esta nueva realidad; sin embargo, la inteligencia artificial no fue abordada en el reciente informe de la Comisión de Productividad de Australia, ni en la respuesta del Gobierno sobre las reformas. Más aún, en la última reforma de ley dada en 2018 sólo ha abordado las infracciones de la propiedad intelectual en línea.

17. Gaudamuz, «La inteligencia...». 
Sin embargo, señala Guadamuz ${ }^{18}$ que conceder la autoría al programador queda reflejada en algunas legislaciones, como la de Hong Kong, India, Irlanda, Nueva Zelanda y el Reino Unido, y que este enfoque se describe claramente en la legislación británica sobre el derecho de autor, en particular en el artículo 9.3 de la Ley de Derechos de Autor, Diseños y Patentes del Reino Unido de 1988 (CDPA), que dispone: «En el caso de una obra literaria, dramática, musical o artística generada por computadora, se considerará que el autor es la persona que realiza los arreglos necesarios para la creación de la obra». Esta ley agregó una disposición que permitía respecto de la autoría de trabajos generados por computadora -en las que el trabajo es generado por una computadora de tal manera que no haya un autor humano- otorgársela a la persona que hace los arreglos necesarios para la creación de la obra. La jurisprudencia actual ha discutido la autoría humana a través del uso de computadoras o el uso de software para generar obras, al igual que CDPA.

Por su parte, la ley canadiense de derechos de autor establece el requisito de que el autor de una obra debe ser ciudadano o sujeto, o una persona que habitualmente reside en un país del tratado. No hay ninguna disposición que aborde los trabajos generados por computadora y ningún caso que trate estos. Sin embargo, el texto de la norma permitiría argumentar que los derechos de autor sólo se aplicarían a obras creadas con la asistencia de computadoras, mas no aplicarían a creaciones de inteligencia artificial generadas por computadora (Ihalainen, 2018: 726).

Si referimos la legislación de la Unión Europea, apunta Ihalainen (2018: 727) que la autoría de la inteligencia artificial parece igualmente dudosa. Más aún, los tribunales de la Unión Europea han cimentado el concepto de creación intelectual del propio autor para referirse a obras en las que el autor debe sellar su toque personal o reflejar su personalidad en el sentido de que expresa sus habilidades creativas de manera original al tomar decisiones libres y creativas. A partir de estas consideraciones, pareciera haber un impulso muy lento en la Unión Europea para desarrollar una la ley sobre derechos de autor que pueda adaptarse a un futuro más automatizado y operado por la inteligencia artificial. El estudio del Comité de Asuntos Jurídicos del Parlamento de la Unión Europea sobre las normas del derecho civil europeo en robótica ha considerado este problema, y recomendó el ajuste de los derechos de autor a la luz de las capacidades nuevas o futuras de los robots autónomos, que se pueda argumentar que se apliquen por igual a la inteligencia artificial incluso en ausencia de una entidad robótica física. Sus recomendaciones en el borrador del informe exigían criterios para la «creación intelectual propia» para obras protegibles producidas por computadoras o robots.

A partir de las diversas posturas señaladas, sin duda destacan dos problemas que la legislación futura tendrá que abordar en esta área: la propiedad y la aplicación de los derechos de autor. Los tribunales también podrían ser una vía para abordar

18. Gaudamuz, «La inteligencia...». 
el problema en el futuro inmediato, como se ha hecho en Nova Productions. ${ }^{19}$ Sin embargo, una legislación propia y específica proporcionaría certeza y un remedio específico para un tema altamente cuestionable.

En este contexto, tampoco debe dejarse de lado que la creación de la inteligencia artificial implica una gran cantidad de aportes monetarios, esfuerzo y creación independiente como parte de un equipo más amplio de desarrolladores. Es necesario establecer firmemente quién es el propietario final de los derechos. Una vez que se ha decidido el propietario final, la aplicación de cualquier derecho en las obras deberá ser restringido y los límites de alcance establecidos, recompensando de un lado y debidamente los esfuerzos de los desarrolladores, pero del otro lado permitiendo que «otros» creen obras, con o sin intervención humana.

\section{Conclusiones}

Deviene en urgente necesidad un profundo análisis en torno a la concesión de derechos a una entidad robótica asociada a la inteligencia artificial, sea que cuente con la característica de corporeidad o no lo para el caso específico de la producción de obras en sus distintos géneros; al tiempo que la determinación sobre dónde deberían estar los derechos o en quién deberían recaer cuando el material producido no tiene ninguna intervención o planificación humana asociada, se convierte en un tema crucial. En ese sentido, la «personalidad» atribuible —cualquiera sea el nombre por el cual se opte- podría otorgarles algunos o todos los derechos sobre las obras creadas. De ahí que ante una creación por el binomio robot e inteligencia artificial, deberá determinarse en primer lugar si éste se encontraría inmersa dentro de alguna calificación de sujeto legal con todo lo que ello implica; y, en segundo lugar, establecer la relación entre una inteligencia artificial y los desarrolladores de software en las diferentes etapas de desarrollo tecnológico, ambos a nuestro parecer puntos de partida mínimos sobre los cuales se podrá continuar para abordar las diversas consideraciones recogidas en el sistema hacia el establecimiento de la titularidad de los derechos de autor sobre la creación de una inteligencia artificial.

Ante una eventual calificación, al parecer quedaría establecida la titularidad del derecho, entendiéndose que ya no habría cuestionamientos en torno a quién sería el propietario o beneficiario de esos derechos, como hasta ahora se viene discutiendo. Aun siendo así, pudiera no estar clara la forma en que la inteligencia artificial usaría o disfrutaría de los ingresos o si es que podría hacerlo. En consecuencia, se avizoraría la posible existencia de un problema adicional respecto de las ganancias.

19. En la sentencia del caso Nova Productions Ltd con Mazooma Games Ltd \& Ors Rev 1, se menciona en el s. 178 que "generado por computadora», en relación con un trabajo, significa que el trabajo es generado por computadora en circunstancias tales que no hay un autor humano del trabajo. 
En la actualidad existen diversos puntos de vista en torno a la inteligencia artificial dentro del campo de la propiedad intelectual. Uno de ellos está a favor de dotarla de personalidad jurídica, bajo una calificación de personalidad limitada o secundaria. El otro considera que, debido a que la inteligencia artificial está controlada por sujetos civiles - personas físicas o jurídicas-, ésta no es suficiente por sí misma como para lograr el estatus de sujeto independiente; más aún, las legislaciones tienen posiciones poco inclusivas en torno a la posible autoría de una inteligencia artificial.

Se ha evidenciado ya en algunos ordenamientos la concesión de derechos a quien, en conjunto con la inteligencia artificial, generó una obra determinada. Sin embargo, consideramos que podrían surgir además otros escenarios, condicionados a las particularidades inherentes a la inteligencia artificial y que harían posible que se reconozca y otorgue la titularidad de la creación y, en consecuencia, de los derechos inherentes de forma total o parcial al propietario o al fabricante. Una segunda opción aborda la denegación de protección la inteligencia artificial, al no reconocerse ni atribuirse algún tipo de personalidad, postura que no compartimos por las consideraciones ya analizadas, pues conllevaría a que estas obras puedan ser utilizadas por cualquiera, en otras palabras, caerían al dominio público desde su creación, hecho que sin duda nos conduciría a otro tipo de cuestionamientos - no obstante la existencia de sectores que consideren que, por razones teleológicas o económicas, no debieran protegerse la obras creadas por la inteligencia artificial-. Asimismo, otro escenario implicaría replantearse los sistemas actuales en torno a la propiedad intelectual, que bien pudieran contemplar formas tuitivas compartidas o conducirlas a formar parte de otros sistemas existentes, como podría ser la competencia desleal. Por último, consideramos también la creación de un sistema de protección ad hoc para estas creaciones, como lo abordó la OMPI.

Creemos, sin embargo, que es indiscutible el hecho de que no sólo la propiedad intelectual deberá replantearse sus contenidos, ya que en el ámbito del sistema de patentes existe una posibilidad aún mayor de colisión entre las leyes de propiedad industrial existentes y los enfoques para generar contenido sin el aporte humano.

En el actual estadio de las cosas, en que las creaciones generadas por la inteligencia artificial podrían ser susceptibles de protección bajo el sistema de propiedad intelectual e incentivar la creación alentando el desarrollo de la robótica, la tutela de la inteligencia artificial requerirá además un replanteamiento del estándar de referencia para la inventiva y potencialmente de todo el sistema actual vigente.

\section{Referencias}

BALALI, Sogol, Ross Sowell, William Smart y Cindy Grimm (2019). «Privacy concerns in robot teleoperation: Does personality influence what should be hidden?». En Miguel Salichs, Shuzhi Sam, Emilia Ivaniva, John-John Cabibihan, Alan Wagner, 
Alvaro Castro y Hongsheng He (editores), Social robotics (pp. 719-729). Cham: Springer.

Balkin, Jack (2015). "The path of robotics law». California Law Review, 6: 45-60. Disponible en https://bit.ly/34TmlH4.

Breazeal, Cynthia (2004). Designing sociable robots. Cambridge: MIT Press.

Boden, Margaret (2011). Creativity and art. Oxford: Oxford Univesity Press.

-. (2015). «Foreword». En Tarek Besold, Marco Schorlemmer y Alan Smaill (editores), Computational creativity research: Towards creative machines (pp. 5-13). París: Atlantis.

-. (2016). AI: Its nature and future. Oxford: Oxford University Press.

Calo, Ryan (2015). «Robotics and the lessons of cyberlaw». California Law Review, 103 (3): 513-563. DOI: $10.2139 /$ ssrn.2402972.

DARLING, Kate (2016). «Extending legal protection to social robots: The effects of anthropomorphism, empathy, and violent behavior towards robotic objects». En Ryan Calo, Michael Froomkin y Ian Kerr (editores), Robot law (pp. 213-231). Cambridge: Edward Elgar Publishing.

ERCILla, Javier (2018). Normas de derecho civil y robótica: Robots inteligentes, personalidad jurídica, responsabilidad civil y regulación. Navarra: Aranzadi.

Groom, Victoria, Leila Takayama, Paloma Ochi y Clifford Nass (2009). «I am my robot: The impact of robot-building and robot form on operators». HRI's Proceedings of the 4th ACM/IEEE international conference on Human robot interaction, 31-36. DOI: 10.1145/1514095.1514104.

Gunkel, David (2018). Robot rights. Cambridge: MIT Press.

Ihalainen, Jani (2018). «Computer creativity: Artificial intelligence and copyright». Journal of Intellectual Property Law \& Practice, 13 (9): 724-728. DOI: 10.1093/jiplp/ jpyo31.

Johnson, Bryan David (2011). Science fiction prototyping: Designing the future with science fiction. Williston: Morgan \& Claypool.

JonEs, Meg y Jason Millar (2017). «Hacking metaphors in the anticipatory governance of emerging technology: The case of regulating robots». En Roger Brownsword, Eloise Scotford y Karen Yeung (editores), The Oxford handbook of law, regulation and technology (pp. 597-619). Oxford: Oxford University Press.

Jordan, John (2015). Robots. Cambridge: MIT Press.

KAC, Eduardo (2001). "The origin and development of robotic art». The International Journal of Research into New Media Technologies, 7 (1): 76-86. DOI: 10.1177/135485650100700108.

-. (2005). Telepresence and bio art: Networking humans, rabbits and robots. Ann Arbor: The University of Michigan Press.

KAPLAN, Jerry (2017). Artificial intelligence: What everyone needs to know. Oxford: Oxford University Press. 
NiLsson, Nils J. (2010). The quest for artificial intelligence: A history of ideas and Achievements. Cambridge: Cambridge University Press.

NourbakHSH, Illah (2013). Robot futures. Cambridge: MIT Press.

-. (2017). Robot fra noi: Le creature intelligenti che stiamo per costruire. Turín: Bollati Boringhieri.

Pagallo, Ugo (2013). The laws of robots: Crimes, contracts, and torts. Amsterdam: Springer.

Penny, Simon (2016). «Robotics and art, computationalism and embodiment». En Damith Herath y Christian Kroos (editores), Robots and art (pp. 47-66). Singapur: Springer.

Richards, Neil M. y William D. Smart (2013). «How should the law think about robots?». SSRN Electronic Journal. DOI: 10.2139/ssrn.2263363.

Riskin, Jessica (2003). «The defecating duck, or, the ambiguous origins of artificial life». Critical Inquiry, 29 (4): 599-633. DOI: 10.1086/377722.

Rueben, Matthew, William Smart, Cindy Grimm y Maya Cakmak (2017). «Privacysensitive robotics». Proceedings of the Companion of the 2017 ACM/IEEE International Conference on Human-Robot Interaction, 425-426.

Rueben, Matthew, Frank Bernieri, Cindy Grimm y William Smart (2019). «Framing effects on privacy concerns about a home telepresence robot». Proceedings of the 2017 ACM/IEEE International Conference on Human-Robot Interaction, 435-444.

Russell, Stuart y Peter Norvig (2004). Inteligencia artificial: Un enfoque moderno. Madrid: Pearson.

SATANOwSKI, Isidro (1954). Derecho intelectual. Buenos Aires: TEA.

Singer, Peter (2011). Practical ethics. Cambridge: Cambridge University Press.

StePhens, Elizabeth y Tara Heffernan (2016). «We have always been robots: The history of robots and art». En Damith Herath y Christian Kroos (editores), Robots and Art (pp. 29-46). Singapur: Springer.

Tiefensee, Christine y Johannes Marx (2015). «Of animals, robots and men». Historical Social Research, 40 (154): 70-91. DOI: 10.12759/hsr.40.2015.4.70-91.

TURKLE, Sherry (2005). The second self: Computers and the human spirit. Cambridge: MIT Pres.

TuRner, Jacob (2019). Robot rules: Regulating artificial intelligence. Londres: Palgrave Macmillan.

Weinzenbaum, Joseph (1987). La frontera entre el ordenador y la mente. Madrid: Pirámide.

Winfield, Alan (2012). Robotics: A very short introduction. Oxford: Oxford University Press.

Wood, Gaby (2007). Edison's eve: A magical history of the quest for mechanical life. Nueva York: Anchor. 
YANISKY, Shlomit (2017). «Generating Rembrandt: Artificial intelligence, copyright, and accountability in the $3 \mathrm{~A}$ Era. The human-like authors are already here. A new model». Michigan State Law Review, 4: 659-726. Disponible en https://bit. ly/35aomMj.

ZHANG, Shujing (2019). «Research on copyright protection of AI creation». Advances in Social Science, Education and Humanities Research, 342: 510-514. DOI: 10.2991/ ielss-19.2019.94.

\section{Sobre la autora}

Ana Karin Chávez Valdivia es abogada. Doctora en Derecho y magíster en Derecho de la Empresa por la Universidad Católica de Santa María, Perú. Magister en Nuevas Tecnologías de la Información y las Comunicaciones, Instituto Europeo Campus Stealle, España. Especialista en Derecho Informático y Gobierno Electrónico por la Universidad Inca Garcilaso de la Vega, Perú. Conciliadora extrajudicial. Directora del Departamento Académico de Ciencias Jurídicas. Docente asociada e investigadora de la Facultad de Derecho de la Universidad La Salle de Arequipa, Perú. Su correo electrónico es achavez@ulasalle.edu.pe. (D) https://orcid.org/oooo-00o2-6453-3119. 


\title{
REVISTA CHILENA DE DERECHO Y TECNOLOGÍA
}

La Revista de Chilena de Derecho y Tecnología es una publicación académica semestral del Centro de Estudios en Derecho Informático de la Facultad de Derecho de la Universidad de Chile, que tiene por objeto difundir en la comunidad jurídica los elementos necesarios para analizar y comprender los alcances y efectos que el desarrollo tecnológico y cultural han producido en la sociedad, especialmente su impacto en la ciencia jurídica.

\author{
EDITOR GENERAL \\ Daniel Álvarez Valenzuela \\ (dalvarez@derecho.uchile.cl) \\ SITIO WEB \\ rchdt.uchile.cl \\ CORREO ELECTRÓNICO \\ rchdt@derecho.uchile.cl \\ LICENCIA DE ESTE ARTÍCULO \\ Creative Commons Atribución Compartir Igual 4.o Internacional
}

\begin{abstract}
La edición de textos, el diseño editorial
y la conversión a formatos electrónicos de este artículo

estuvieron a cargo de Tipográfica

(www.tipografica.io).
\end{abstract}

